\title{
The stellar populations of E and S0 galaxies as seen with SAURON
}

\author{
Harald Kuntschner ${ }^{1}$, Eric Emsellem ${ }^{2}$, Roland Bacon ${ }^{2}$, Martin Bureau ${ }^{3}$, \\ Michele Cappellari ${ }^{4}$, Roger L. Davies ${ }^{3}$, Tim de Zeeuw ${ }^{4}$, Jesús \\ Falcón-Barroso ${ }^{4}$, Davor Krajnović ${ }^{3}$, Richard M. McDermid ${ }^{4}$, Reynier F. \\ Peletier ${ }^{5}$, and Marc Sarzi ${ }^{3}$ \\ 1 Space Telescope European Coordinating Facility, Garching, Germany \\ hkuntsch@eso.org \\ 2 Centre de Recherche Astronomique de Lyon, Lyon, France \\ 3 University of Oxford, Oxford, UK \\ ${ }^{4}$ Leiden Observatory, Leiden, The Netherlands \\ 5 Kapteyn Astronomical Institute, Groningen, The Netherlands
}

Summary. We present selected results from integral-field spectroscopy of 48 earlytype galaxies observed as part of the SAURON survey. Maps of the $\mathrm{H} \beta, \mathrm{Fe} 5015, \mathrm{Mg} b$ and Fe5270 indices in the Lick/IDS system were derived for each of the survey galaxies. The metal line strength maps show generally negative gradients with increasing radius roughly consistent with the morphology of the light profiles. Remarkable deviations from this general trend exist, particularly the $\mathrm{Mg} b$ isoindex contours appear to be flatter than the isophotes of the surface brightness for about $40 \%$ of our galaxies without significant dust features. Generally these galaxies exhibit significant rotation. We infer from this that the fast-rotating component features a higher metallicity and/or an increased $\mathrm{Mg} / \mathrm{Fe}$ ratio as compared to the galaxy as a whole.

We also use the line strengths maps to compute average values integrated over circular apertures of one effective radius, and derive luminosity weighted ages and metallicities. The lenticular galaxies show a wide range in age and metallicity estimates, while elliptical galaxies tend to occupy regions of older stellar populations.

\section{The SAURON survey}

We are carrying out a survey of the dynamics and stellar populations of 72 representative nearby early-type galaxies and spiral bulges based on measurements of the two-dimensional kinematics and line strengths of stars and gas with SAURON, a custom-built panoramic integral-field spectrograph for the William Herschel Telescope, La Palma (Bacon et al. [1]). The goals and objectives of the SAURON survey are described in de Zeeuw et al. [2], which also presents the definition of the sample. The full maps of the stellar kinematics for the 48 elliptical (E) and lenticular (S0) galaxies are given in Emsellem et al. [3]. The morphology and kinematics of the ionised gas emission are presented in Sarzi et al. [4]. Here we summarize selected results of the absorption line strength measurements, which are described more fully in Kuntschner et al. [5]. 


\section{$2 \mathrm{Mg} b$ isoindex contours versus isophotes}

One of the most interesting aspects of integral-field spectroscopy is the capability to identify two-dimensional structures. For the first time we can use this in connection with line strength indices and compare isoindex contours with the isophotal shape. One might expect the index to follow the light in slowly-rotating giant elliptical galaxies, since the stars are dynamically well mixed. However, one could imagine that dynamical substructures with significantly different stellar populations could leave a signature in line strength maps which are sensitive to e.g., metallicity.

The Mg $b$ index is the most stringent feature in our survey and potential differences between isoindex contours and isophotes should be most apparent. Indeed we find a number of galaxies in our survey where the isoindex contours clearly do not follow the isophotes, e.g., NGC 4570 and NGC 4660 (see Fig. 1). Both galaxies show fast-rotating components along the direction of enhanced $\mathrm{Mg} b$ strength. In order to further study such a possible connection we determine the best fitting simple, elliptical model for each of the reconstructed images and $\mathrm{Mg} b$ maps in our survey (excluding all galaxies with significant dust absorption). For an example of this procedure see Fig. 2.
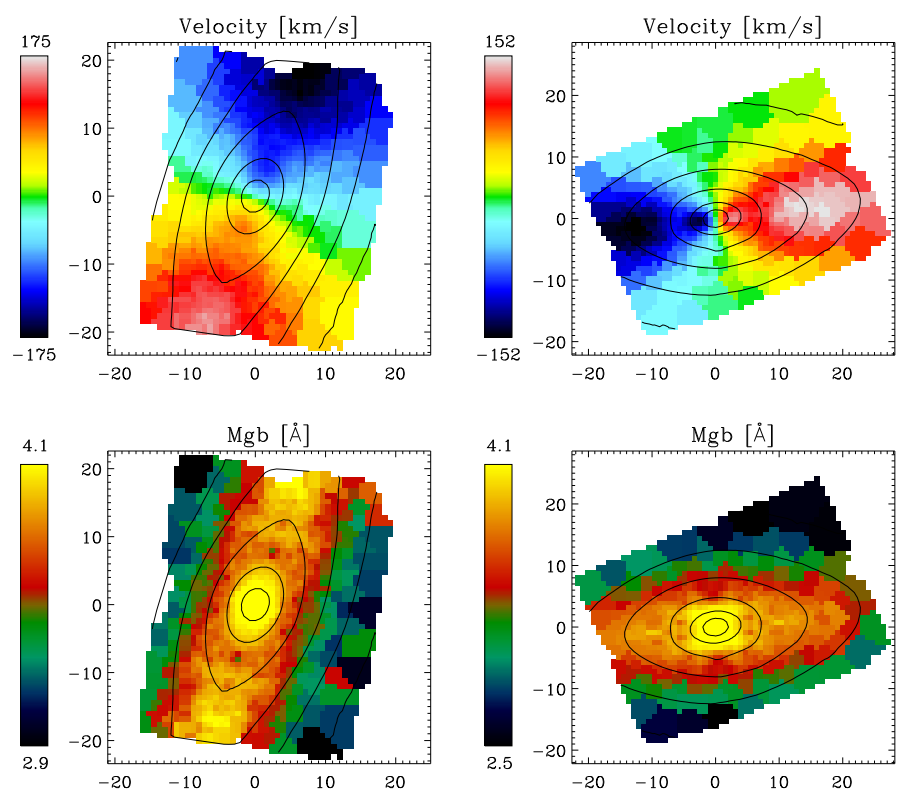

Fig. 1. The velocity maps and $\mathrm{Mg} b$ maps are shown for NGC 4570 (left) and NGC 4660 (right). Both galaxies show fast-rotating components along the direction of enhanced $\mathrm{Mg} b$ strength. For presentation purposes the $\mathrm{Mg} b$ maps are symmetrized. The $\mathrm{x}$ - and $\mathrm{y}$-axis are given in arcsec; North is up and East to the left. 

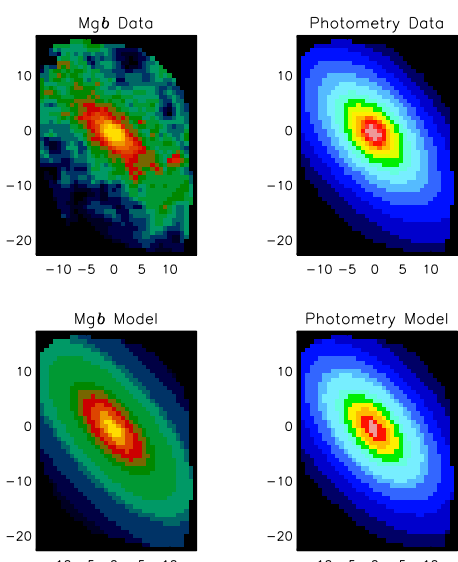

Fig. 2. Top row: The interpolated $\mathrm{Mg} b$ map and the reconstructed image of NGC 3377. Bottom row: The elliptical models with constant position angle and ellipticity fitted to the $\operatorname{Mg} b$ map and the reconstructed image. The best fitting ellipticity for the isophotes and the $\mathrm{Mg} b$ map is $0.473 \pm 0.003$ and $0.573 \pm 0.022$, respectively. The $\mathrm{x}$ - and $\mathrm{y}$-axis are given in arcsec; North is up and East to the left.

The final results of the ellipse fitting are shown in Fig. 3. Applying a $2 \sigma$ error cut, 16 out of 41 galaxies appear to have more flattened $\mathrm{Mg} b$ contours than the isophotes. Most of these galaxies show a high degree of rotational support in the direction of enhanced $\operatorname{Mg} b$ strength. Thus, the flattened $\operatorname{Mg} b$ distribution suggests that the fast-rotating components in these galaxies exhibit a stellar population different from the main body. The enhanced $\operatorname{Mg} b$ strength can be interpreted to first order as higher metallicity and/or increased $[\mathrm{Mg} / \mathrm{Fe}]$ ratio.

\section{Stellar populations}

In order to provide a global measurement of the stellar populations for each galaxy we derive a central averaged spectrum from all data available within one effective radius $R_{e}$. Since our line strength maps do not always cover the full area of one effective radius we apply aperture corrections. However, even the galaxies with the smallest coverage (NGC 4486 and NGC5846) feature line strength data out to about $30 \%$ of $R_{e}$ (corresponds to a field coverage of $\left.\approx 60^{\prime \prime}\right)$ and corrections remain small $(<8 \%)$. The median coverage of the line strength maps in our survey is $0.8 R_{e}$

Estimates of the luminosity weighted age and metallicity of early-type galaxies can be inferred from index-index diagrams. Generally a metallicity sensitive index is plotted against an age sensitive index. In order to minimize the influence of abundance ratio variations on our metallicity estimates 


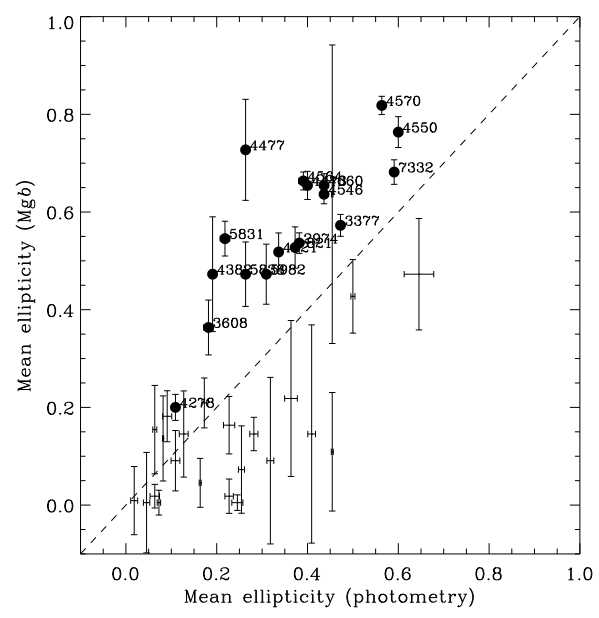

Fig. 3. Comparison of the average ellipticity of constant $\operatorname{Mg} b$ strength with the best fitting elliptical model of the isophotes. Errors are evaluated by a MonteCarlo simulation. All galaxies which are more than $2 \sigma$ above the one-to-one line are indicated by filled circles and their NGC numbers.

we use a composite index $[\mathrm{MgFe} 50]^{\prime}$. This index is constructed such that a mean metallicity is measured rather than a metallicity biased by non-solar abundance ratios.

Fig. 4 shows a $\mathrm{H} \beta$ vs $[\mathrm{MgFe} 50]^{\prime}$ diagram. Overplotted are the model predictions from Thomas, Maraston \& Bender [6]. The lenticular galaxies in our sample show a wide range in age and metallicity estimates. The elliptical galaxies however, tend to occupy regions of older stellar populations. There is weak evidence that ellipticals in the field may on average have experienced some "frosting" of young stars yielding lower luminosity weighted age estimates. It is worth noticing that luminosity weighted metallicities rarely exceed solar metallicity when taking an average over one effective radius.

\section{Conclusions}

The results presented here are the outcome of the first comprehensive survey of the line strength distributions of nearby early-type galaxies with an integral-field spectrograph. This data set demonstrates that many nearby early-type galaxies display a significant and varied structure in their line strength properties. This structure can be of subtle nature as seen in the deviations of $\mathrm{Mg} b$ isoindex contours compared to the isophotes of galaxies.

The $2 \mathrm{D}$ coverage of the line strength allows us to connect the stellar populations with the kinematical structure of the galaxies and thus further our knowledge of the star-formation and assembly history of early-type galaxies. 


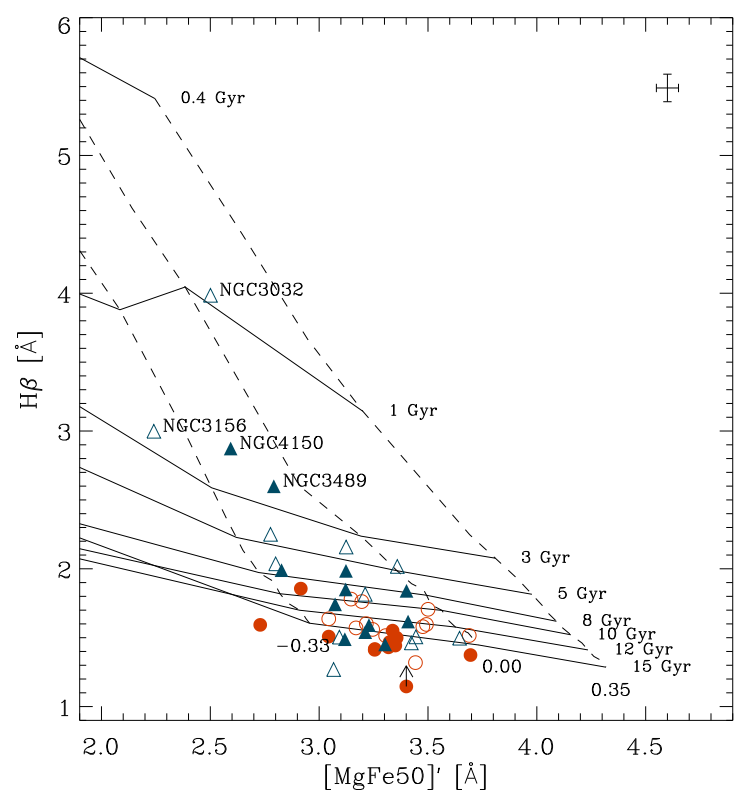

Fig. 4. Central, one $R_{e}$ aperture $\mathrm{H} \beta$ versus $[\mathrm{MgFe} 50]^{\prime}$ line strength measurements. The filled and open circles are cluster and field ellipticals, respectively; filled and open triangles represent cluster and field S0s, respectively. Overplotted are models by Thomas, Maraston \& Bender [6]. The error bar in the right upper corner gives the mean offset error to the Lick/IDS system.

\section{References}

1. Bacon R., Copin Y., Monnet G., Miller B. W., Allington-Smith J. R., Bureau M., Carollo M. C., Davies R. L., Emsellem E., Kuntschner H., Peletier R. F., Verolme E. K., de Zeeuw P. T., MNRAS, 326, 23 (2001)

2. de Zeeuw P. T., Bureau M., Emsellem E., Bacon R., Carollo C. M., Copin Y., Davies R. L., Kuntschner H., Miller B. W., Monnet G., Peletier R. F., Verolme E. K., MNRAS, 329, 513 (2002)

3. Emsellem E., Cappellari M., Peletier R. F., McDermid R. M., Bacon R., Bureau M., Copin Y., Davies R. L., Krajnović D., Kuntschner H., Miller B. W., de Zeeuw P. T., MNRAS, 352, 721 (2004)

4. Sarzi M., Falcón-Barroso J., Davies R. L., Bacon R., Bureau M., Cappellari M., de Zeeuw P. T., Emsellem E., Fathi K., Krajnović D., Kuntschner H., McDermid R. M., Peletier R. F., 2006, MNRAS, accepted, astro-ph/0511307

5. Kuntschner H., Emsellem E., Bacon R., Bureau M., Cappellari M., Davies R. L., de Zeeuw P. T., Falcón-Barroso J., Fathi K., Krajnović D., McDermid R. M., Peletier R. F., Sarzi M., 2006, MNRAS, submitted

6. Thomas D., Maraston C., Bender R., MNRAS, 339, 897 (2003) 